\title{
Bone density and pain response following intensity-modulated radiotherapy versus three-dimensional conformal radiotherapy for vertebral metastases - secondary results of a randomized trial
}

\author{
Tanja Sprave ${ }^{1,3}$, Vivek Verma ${ }^{2}$, Robert Förster ${ }^{1,3,4}$, Ingmar Schlampp ${ }^{1,3}$, Katharina Hees ${ }^{2,5}$, Thomas Bruckner ${ }^{2,5}$, \\ Tilman Bostel ${ }^{1}$, Rami Ateyah El Shafie ${ }^{1,3}$, Thomas Welzel' ${ }^{1}$, Nils Henrik Nicolay ${ }^{1,3,6}$, Jürgen Debus ${ }^{1,3}$ \\ and Harald Rief ${ }^{1,3 *}$ (i)
}

\begin{abstract}
Background: This was a prespecified secondary analysis of a randomized trial that analyzed bone density and pain response following fractionated intensity-modulated radiotherapy (IMRT) versus three-dimensional conformal radiotherapy (3DCRT) for palliative management of spinal metastases.

Methods/materials: Sixty patients were enrolled in the single-institutional randomized exploratory trial, randomly assigned to receive IMRT or 3DCRT (30 Gy in 10 fractions). Along with pain response (measured by the Visual Analog Scale (VAS) and Chow criteria), quantitative bone density was evaluated at baseline, 3, and 6 months in both irradiated and unirradiated spinal bodies, along with rates of pathologic fractures and vertebral compression fractures.

Results: Relative to baseline, bone density increased at 3 and 6 months following IMRT by a median of 24.8\% and $33.8 \%$, respectively $(p<0.01$ and $p=0.048)$. These figures in the 3DCRT cohort were $18.5 \%$ and $48.4 \%$, respectively ( $p<0.01$ for both). There were no statistical differences in bone density between IMRT and 3DCRT at 3 ( $p=0.723$ ) or 6 months $(p=0.341)$. Subgroup analysis of osteolytic and osteoblastic metastases showed no differences between groups; however, mixed metastases showed an increase in bone density over baseline in the IMRT (but not 3DCRT) arm. The 3-month rate of the pathological fractures was $15.0 \%$ in the IMRT arm vs. $10.5 \%$ in the 3DCRT arm. There were no differences in pathological fractures at $3(p=0.676)$ and $6(p=1.000)$ months. The IMRT arm showed improved VAS scores at $3(p=0.037)$ but not 6 months $(p=0.430)$. Using Chow criteria, pain response was similar at both $3(p=0.395)$ and $6(p=0.732)$ months.
\end{abstract}

Conclusions: This the first prospective investigation evaluating the impact of IMRT vs. 3DCRT on bone density. Along with pain response and pathologic fracture rates, significant rises in bone density after 3 and 6 months were similar in both cohorts. Future randomized investigations with larger sample sizes are recommended.

Trial registration: NCT, NCT02832830. Registered 14 July 2016

Keywords: Bone metastases, Spine, Intensity-modulated radiation therapy, Bone density, Palliative radiotherapy

\footnotetext{
* Correspondence: harald.rief@gmx.at

${ }^{1}$ University Hospital of Heidelberg, Department of Radiation Oncology, Im

Neuenheimer Feld 400, 69120 Heidelberg, Germany

${ }^{3}$ Heidelberg Institute of Radiation Oncology (HIRO), Im Neuenheimer Feld

280, 69120 Heidelberg, Germany

Full list of author information is available at the end of the article
}

(c) The Author(s). 2018 Open Access This article is distributed under the terms of the Creative Commons Attribution 4.0 International License (http://creativecommons.org/licenses/by/4.0/), which permits unrestricted use, distribution, and reproduction in any medium, provided you give appropriate credit to the original author(s) and the source, provide a link to the Creative Commons license, and indicate if changes were made. The Creative Commons Public Domain Dedication waiver (http://creativecommons.org/publicdomain/zero/1.0/) applies to the data made available in this article, unless otherwise stated. 


\section{Introduction}

Spinal metastases, which occur in up to $40 \%$ of advancedstage cancer patients, can be a major source of symptomatic burden and quality of life detriment [1]. These include not only pain and immobility, but also neurological deficits and risk of pathological fractures. Historically, conventionally fractionated three-dimensional conformal radiotherapy (3DCRT) has been the technique of choice to palliate these cases $[2,3]$. However, the advancement of technologies such as intensity-modulated radiation therapy (IMRT) allows for safer dose-escalation by means of higher conformality, image guidance, and decreased doses to nearby organs-at-risk (OARs).

Because much of current research on spinal metastases involves stereotactic radiotherapy (achieved in 5 or fewer fractions), fractionated IMRT has remained an understudied option for these cases. Although stereotactic radiotherapy may be accomplished by inverse-planned IMRT, volumetric modulated arc therapy (VMAT), or TomoTherapy techniques, fractionated IMRT (most commonly 30 Gy in 10 fractions as in this study), which can also be performed with any of the aforementioned techniques, has largely been overshadowed to date and thus deserves further study.

There are known serious adverse events associated with spinal irradiation, such as decreases in bone density potentially resulting in vertebral compression fractures (VCFs). There are no randomized data evaluating these parameters in IMRT versus 3DCRT to date. This was a prespecified secondary analysis of a randomized trial, which evaluated bone density and pain response following IMRT versus conventional 3DCRT as part of palliative management of painful spinal metastases.

\section{Materials and methods}

\section{Trial design and participants}

The randomized trial, registered on clinicaltrials.gov (NCT02832830), was approved by the Heidelberg University Independent Ethics Committee (Nr. S-238/2016). Details of the study design have been published previously [4]. The primary endpoint of this randomized, single-institutional, pilot trial was 3-month RT-induced toxicity following delivery of $30 \mathrm{~Gy}$ in 10 fractions of image-guided IMRT versus conventional 3DCRT in patients with previously untreated spinal metastases. All patients had an established indication for RT, including pain and/or neurological deficits. The present study was a prespecified secondary analysis of bone density, as well as pain response and rates of pathologic fracture and VCF.

A block randomization approach (block size of 6) was used to ensure that the two groups were balanced. In addition to the above, inclusion criteria were ages 18-85, a Karnofsky performance score $\geq 50$, and ability to provide written informed consent. Exclusion criteria were subjects with significant neurological or psychiatric disorders precluding informed consent, previous RT to the given irradiation site, or multiple myeloma or lymphoma histology. Number or location of metastases were not specific criteria for inclusion or exclusion, nor was the presence of spinal cord compression.

\section{Assessment of endpoints}

Per protocol, bone density in irradiated and non-irradiated vertebral bodies, other pathologic vertebral fractures, and VCFs were assessed at baseline and at 3 and 6 months after RT. Bone density was assessed with the Syngo Osteo CT workstation in manually selected regions of interest. Hounsfield units (HU) were used for bone density measurements. The Siemens Somatom Sensation Open (Siemens, Erlangen, Germany) scanner was used for all CT examinations. Measurements were carried out at the appropriate site by a single physician in light of interobserver bias. During the observation period, because most participants received anti-osteoresoptive treatment, changes in bone density in unaffected vertebrae were also measured.

Pathologic fractures were diagnosed by experienced radiologists by means of CT and/or MRI imaging by comparing to baseline imaging tests. New fractures were, by definition, not present on initial imaging, whereas progressive fractures referred to visibly increasing size and/ or number of fracture gaps, dislocation of fracture fragments, or increasing sintering of the VCF. A VCF was defined as the reduction of the vertebral body height by more than $20 \%$. Each of these was grouped under the term of "pathologic fractures".

In addition to evaluating neuropathic pain, overall pain response to RT was quantified by the visual analog scale (VAS), measured at the irradiated region prior to, immediately following, and at 3 and 6 months after RT. Pain response was designated as complete response (CR), partial response $(\mathrm{PR})$, pain progression $(\mathrm{PP})$, and intermediate pain (IP) according to the International Bone Consensus response categories by Chow et al. [5] Complete response $(\mathrm{CR})$ was defined as no pain (VAS = 0 ) after 3 months and partial response (PR) as an improvement by at least two points after 3 and 6 months. $\mathrm{CR}$ referred to VAS $=0$ with no concurrent increase in analgesic intake (stable or reducing analgesics in daily oral morphine equivalents). PR was pain reduction of 2 or more without increase in analgesics, or analgesic reduction of at least $25 \%$ from baseline without an increase in pain. PP was defined as increase in pain score of $\geq 2$ above baseline with stable oral morphine equivalents, or an increase of $25 \%$ or more in the latter with the pain score stable or 1 point above baseline. Any response not covered by the CR, PR or PP definitions was 
Table 1 Baseline characteristics of randomly assigned participants

\begin{tabular}{|c|c|c|c|c|c|}
\hline & \multicolumn{2}{|c|}{ IMRT group $n=30$} & \multicolumn{2}{|c|}{$3 \mathrm{D}$ group $n=30$} & \multirow[t]{2}{*}{$p$-value } \\
\hline & $n$ & $\%$ & $n$ & $\%$ & \\
\hline Age (years) & & & & & 0.219 \\
\hline Mean (SD) & $66,1(10,5)$ & & $62,5(11,8)$ & & \\
\hline Karnofsky-Perfomance Status & & & & & 0.283 \\
\hline Mean (SD) & $64,9(9,32)$ & & $61,3(9,7)$ & & \\
\hline Gender & & & & & 0.795 \\
\hline Male & 17 & 56,7 & 16 & 53,3 & \\
\hline Female & 13 & 43,3 & 14 & 46,7 & \\
\hline Weight (kg, SD) & $75,8(14,9)$ & & $76,2(19,4)$ & & 0.929 \\
\hline Height $(\mathrm{cm}, \mathrm{SD})$ & $171,6(8,8)$ & & $172,2(8,6)$ & & 0.790 \\
\hline Body mass index (BMI) & & & & & 0.960 \\
\hline Mean (SD) & $25,7(4,4)$ & & $25,6(5,7)$ & & \\
\hline \multicolumn{6}{|l|}{ Primary site } \\
\hline Lung cancer & 11 & 36,7 & 16 & 53,3 & \\
\hline ABreast cancer & 7 & 23,3 & 6 & 20 & \\
\hline Prostata cancer & 6 & 20 & 1 & 3,3 & \\
\hline Other & 6 & 20 & 7 & 23,3 & \\
\hline \multicolumn{6}{|l|}{ Volume of metastases at baseline } \\
\hline Mean size $\left(\mathrm{mm}^{3}\right)$ & 30 & 1166,6 & 30 & & 0.191 \\
\hline Localization metastases & & & & & 0.261 \\
\hline Cervical & 4 & 13,3 & 5 & 16,7 & \\
\hline Thoracic & 15 & 50 & 15 & 50 & \\
\hline Lumbar & 11 & 36,7 & 7 & 23,3 & \\
\hline Sacrum & 0 & 0 & 3 & 10 & \\
\hline Number metastases & & & & & 0.140 \\
\hline 1 metastase & 17 & 56,7 & 10 & 33,3 & \\
\hline 2 metastases & 4 & 13,3 & 9 & 30 & \\
\hline 3 metastases & 9 & 30 & 11 & 36,7 & \\
\hline \multicolumn{6}{|l|}{ Distant metastases at baseline } \\
\hline Viszeral & 14 & 46,7 & 10 & 33,3 & 0.292 \\
\hline Lung & 7 & 23,3 & 6 & 20 & 0.754 \\
\hline Brain & 4 & 13,3 & 5 & 16,7 & 0.718 \\
\hline Tissue & 5 & 16,7 & 5 & 16,7 & 1.000 \\
\hline Hormontherapy & 12 & 40 & 6 & 20 & 0.091 \\
\hline Immuntherapy & 4 & 13,3 & 5 & 16,7 & 0.718 \\
\hline Chemotherapy & 14 & 46,7 & 20 & 66,7 & 0.118 \\
\hline Surgery & 18 & 60 & 13 & 43,3 & 0.196 \\
\hline Neurological deficit at baseline & 4 & 13,3 & 3 & 10 & 0.688 \\
\hline Bisphosphonate at baseline & 13 & 43,3 & 7 & 23,3 & 0.100 \\
\hline Orthopedic corset at baseline & 9 & 30 & 10 & 33,3 & 0.781 \\
\hline \multicolumn{6}{|l|}{ Medication at baseline } \\
\hline Sleeping medication & 5 & 16,7 & 2 & 6,7 & 0.228 \\
\hline Psychiatric medication & 9 & 30 & 6 & 20 & 0.371 \\
\hline Opiate & 20 & 66,7 & 17 & 56,7 & 0.426 \\
\hline
\end{tabular}


Table 1 Baseline characteristics of randomly assigned participants (Continued)

\begin{tabular}{|c|c|c|c|c|c|}
\hline & \multicolumn{2}{|c|}{ IMRT group $n=30$} & \multicolumn{2}{|c|}{$3 \mathrm{D}$ group $n=30$} & \multirow[t]{2}{*}{$p$-value } \\
\hline & $n$ & $\%$ & $n$ & $\%$ & \\
\hline NSAID & 23 & 76,7 & 19 & 63,3 & 0.260 \\
\hline
\end{tabular}

Explanation: Others: carcinoma of unknown primary (CUP), gastrointestinal stromal tumor (GIST), melanoma, mesothelioma, pancreatic cancer, renal cancer. Abbreviations: NSAID nonsteroidal inflammatory drug

called "stable pain". Responders were defined as having $\mathrm{CR}$ or PR, and non-responders as having PP or IP.

Exploratory analysis of overall survival (OS) was performed and defined as the time from initial diagnosis until death or censored at last contact.

\section{Radiotherapy}

CT simulation was performed with custom immobilization using Aquaplast ${ }^{\ominus}$ (Aquaplast Corporation, Wyckoff, NJ, USA) head masks for cervical spine cases and Wingstep/ Prostep ${ }^{\oplus}$ (Elekta, Stockholm, Sweden) devices for thoracolumbar cases. In addition to OARs (dose constraints for which were per QUANTEC recommendations), the clinical target volume (CTV) was delineated on the planning CT and encompassed the affected vertebral body [6]. The planning target volume (PTV) was an isotropic $1 \mathrm{~cm}$ expansion of the CTV and was to be covered by the $90 \%$ isodose line. The prescription dose for both cohorts was $30 \mathrm{~Gy}$ in 10 fractions.

The IMRT group received image-guided (mega- or kilovoltage cone beam computed tomography) RT by means of step-and-shoot IMRT, VMAT (Elekta Versa HD accelerator), or helical TomoTherapy (Accuray Inc., Madison, WI). The 3DCRT cohort was most commonly delivered with two or three anteroposterior $6 \mathrm{MV}$ individuallyformed beams. Position verification was applied by weekly kilo-voltage CT and before each fraction by comparing orthogonal portal images with digitally reconstructed radiographs from the planning CT.

\section{Statistical analysis}

Complete details regarding statistical analysis are presented elsewhere [4]. Owing to the exploratory nature of this study, a complete power calculation was not possible; however, with 30 patients in each group, it was possible to detect a standardized mean-value effect of 0.8 with $80 \%$ power at a significance level of 0.05 .

All variables were analyzed descriptively by tabulation of the measures of the empirical distributions. According to the scale level of the variables, means (Hodges-Lehmann estimates) and standard deviations or absolute and relative frequencies, respectively, were reported. Additionally, for variables with longitudinal measurements, the time courses of individual patients and summarized by treatment groups. Descriptive $p$-values of the corresponding statistical tests comparing the treatment groups were given. Analysis of covariance (ANOVA) with repeated measurements, with treatment group as a

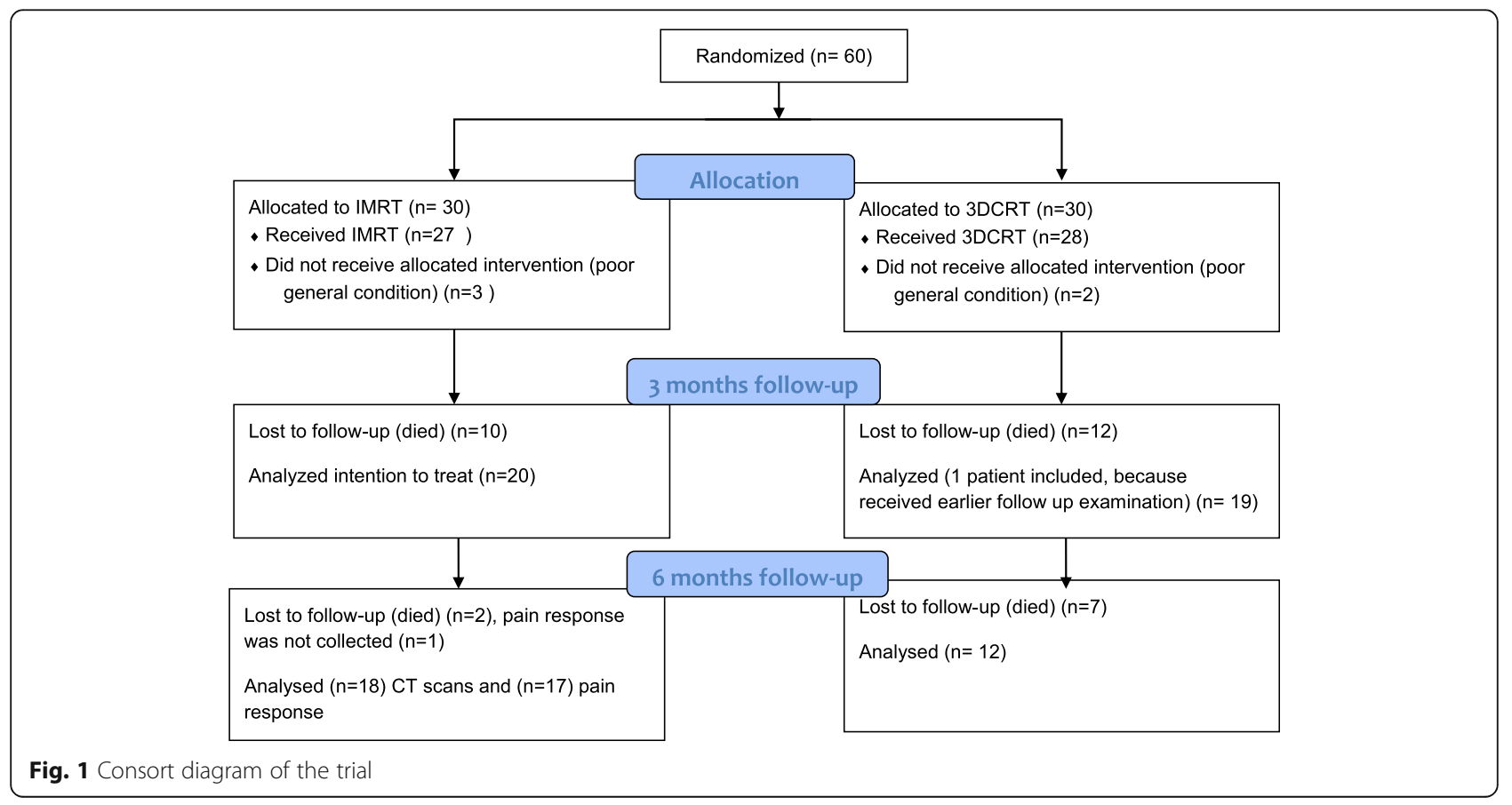




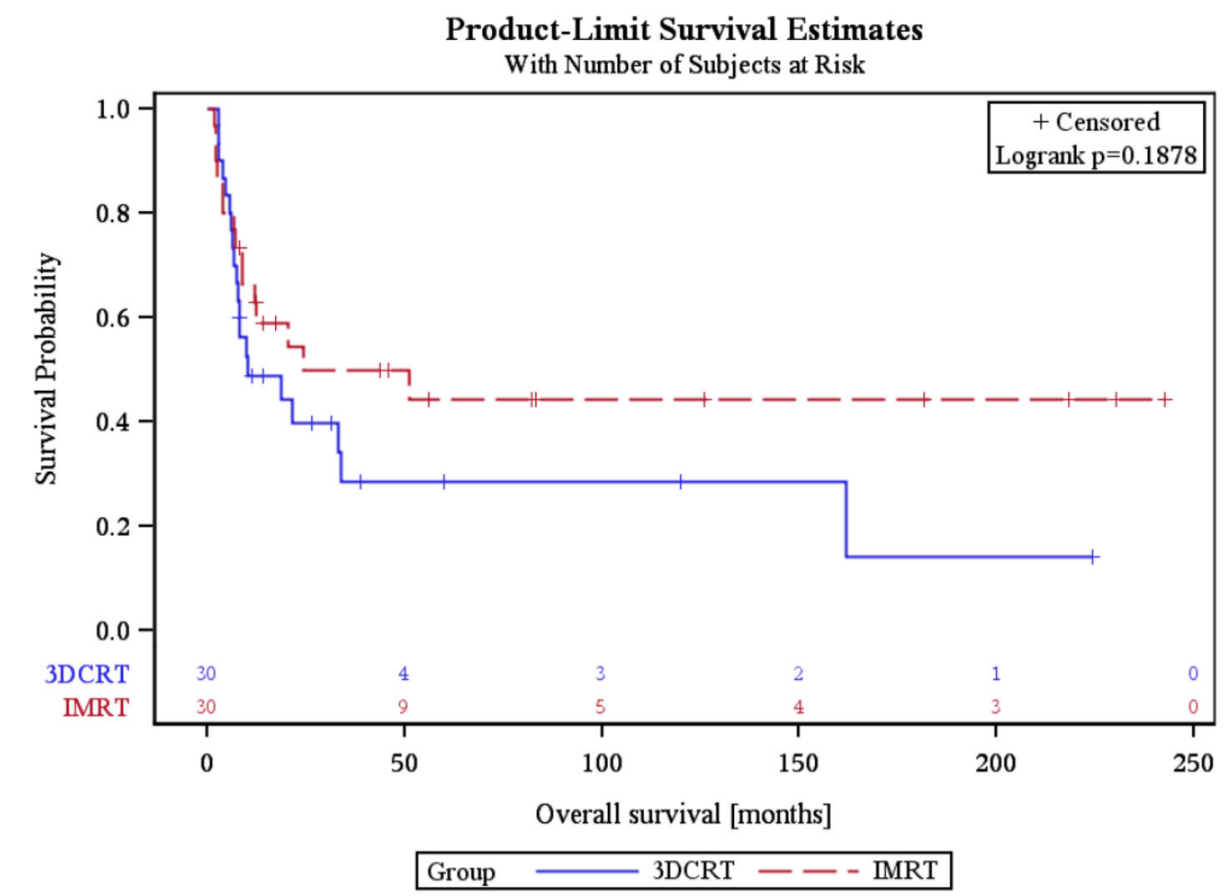

Fig. 2 Overall survival of both arms

factor, and pain medication as covariates, were done. The Wilcoxon rank-sum test was used to detect possible differences between groups after 3 and 6 months. All statistical analyses were done using SAS software Version 9.4 or higher (SAS Institute, Cary, NC, USA).

\section{Funding source}

The sponsors of the study had no role in study design, data analysis, data interpretation and wording of the report. The corresponding author (HR) had full access to the entire data of the study and had final responsibility regarding the decision to submit for publication.

\section{Results}

From November 2016 to May 2017, 60 patients were randomized. No patients were excluded post randomization. Baseline characteristics were balanced between the two treatment arms (Table 1, as previously reported) [7].

Although all surviving patients completed all assessments, not all patients survived by the three and six month time periods. Within the first 3 months, 10 patients

Table 2 Bone density of both groups in metastatic bone before RT (baseline), as well as 3 and 6 months after RT

\begin{tabular}{|c|c|c|c|c|c|c|c|c|c|c|c|}
\hline & \multicolumn{2}{|c|}{ IMRT group } & \multirow[b]{2}{*}{ SD } & \multirow{2}{*}{$\begin{array}{l}\text { Within group } \\
p \text {-value }\end{array}$} & \multicolumn{2}{|c|}{ 3DCRT group } & \multirow[b]{2}{*}{ SD } & \multirow{2}{*}{$\begin{array}{l}\text { Within group } \\
p \text {-value }\end{array}$} & \multicolumn{2}{|c|}{ Differences between groups } & \multirow[b]{2}{*}{$p$-value } \\
\hline & $\bar{n}$ & Median & & & $n$ & Median & & & $\overline{\mathrm{HL}}$ & $95 \% \mathrm{Cl}$ & \\
\hline \multicolumn{12}{|l|}{ All metastases } \\
\hline $\begin{array}{l}\mathrm{HU} \\
\text { Baseline }\end{array}$ & 30 & 258.5 & 183.3 & & 30 & 195.0 & 125.4 & & 62 & $5.0-126.0$ & 0.037 \\
\hline $\mathrm{HU} \mathrm{T} 2$ & 20 & 419.3 & 232.7 & & 19 & 300.0 & 165.7 & & -59.5 & $-194.0-59.0$ & 0.232 \\
\hline HU T3 & 18 & 416.8 & 277.7 & & 12 & 454.0 & 185.4 & & 61.5 & $-146.0-229$ & 0.641 \\
\hline \multicolumn{12}{|l|}{3 months } \\
\hline HU T0-T2 & 20 & 90.5 & 134.2 & $<0.01$ & 19 & 35.0 & 87.1 & $<0.01$ & -25.0 & $-86.0-42.0$ & 0.407 \\
\hline HU TO-T2 (\%) & 20 & 24.8 & 51.0 & $<0.01$ & 19 & 18.5 & 38.7 & $<0.01$ & -4.5 & $-25.5-21.9$ & 0.723 \\
\hline \multicolumn{12}{|l|}{6 months } \\
\hline HU TO-T3 & 18 & 124.0 & 166.0 & 0.023 & 12 & 132.0 & 157.7 & $<0.01$ & 59.0 & $-73.0-193.0$ & 0.330 \\
\hline HU T0-T3 (\%) & 18 & 33.8 & 61.6 & 0.048 & 12 & 48.4 & 70.7 & $<0.01$ & 34.0 & $-20.3-91.1$ & 0.341 \\
\hline
\end{tabular}

The results were presented by absolute and relative values (\%) of $\mathrm{HU}$ within and between groups as median (Hodges-Lehmann estimate) and IQR 
Table 3 The subgroup analysis of the bone density ( $H U=$ Hounsfield units) in metastatic bone before RT (baseline), as well as 3 and 6 months after RT

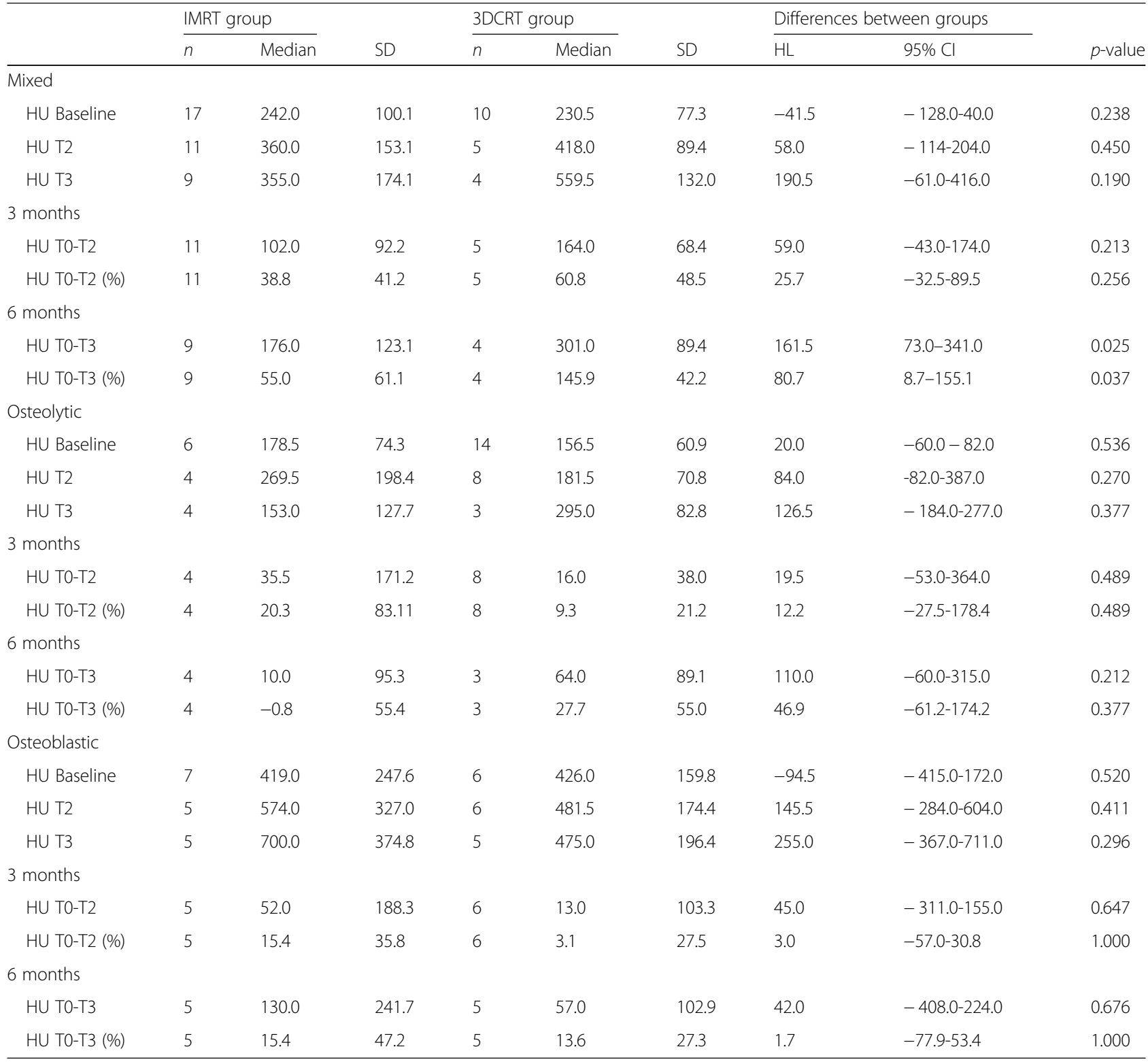

The results were presented by absolute and relative values (\%) of $\mathrm{HU}$ within and between groups as median (Hodges-Lehmann estimate) and IQR. Abbreviations: HU Hounsfield units, IQR interquartile range, TO baseline, T2 3 months, T3 6 months, TO-T2 difference in baseline minus 3 months, TO-T3 difference in baseline minus 6 months

Table 4 Results of pathological fractures of both groups

\begin{tabular}{|c|c|c|c|c|c|c|c|}
\hline & \multirow{3}{*}{$\begin{array}{l}\text { IMRT group } \\
n\end{array}$} & \multirow{3}{*}{$\begin{array}{l}n /(\%) \\
\text { No }\end{array}$} & \multirow[b]{3}{*}{ Yes } & \multicolumn{2}{|c|}{ 3DCRT group } & \multirow[b]{3}{*}{ Yes } & \multirow{3}{*}{$\begin{array}{l}\text { Differences between groups } \\
p \text {-value }\end{array}$} \\
\hline & & & & $n$ & $n /(\%)$ & & \\
\hline & & & & & No & & \\
\hline Baseline & 30 & $29(96.7 \%)$ & $1(3.3 \%)$ & 30 & $26(86.7 \%)$ & $4(13.3 \%)$ & 0.161 \\
\hline 3 months & 20 & 17 (85.0\%) & $3(15 \%)$ & 19 & 17 (89.5\%) & $2(10.5 \%)$ & 0.676 \\
\hline 6 months & 18 & 15 (83.3\%) & $3(16.7 \%)$ & 12 & 10 (83.3\%) & $2(16.7 \%)$ & 1.000 \\
\hline
\end{tabular}


Table 5 Response according to Brief Pain Inventory score at 3 and 6 months in the per-protocol cohort

\begin{tabular}{llllll}
\hline & IMRT group $n=20$ & \multicolumn{3}{l}{ 3DCRT group $n=19$} & \\
\hline After 3 months & $\mathrm{n}$ & $\%$ & $\mathrm{n}$ & $\%$ & $p$-value \\
CR & 10 & 50 & 5 & 26,3 & 0.395 \\
PR & 4 & 20 & 4 & 20,1 & \\
PP & 1 & 5 & 3 & 15,8 & \\
IP & 5 & 25 & 7 & 36,8 & \\
Responders & 14 & 70 & 9 & 47,4 & 0.151 \\
Non-responders & 6 & 30 & 10 & 52,6 & \\
& IMRT group $n=17$ & 3DCRT group $n=12$ &
\end{tabular}

After 6 months

$\begin{array}{llllll}\text { CR } & 7 & 41,2 & 3 & 25 & 0.732 \\ \text { PR } & 5 & 29,4 & 4 & 33,3 & \\ \text { PP } & 2 & 11,8 & 3 & 25 & \\ \text { IP } & 3 & 17,7 & 2 & 16,7 & \\ \text { Responders } & 12 & 70,8 & 7 & 58,3 & 0.494 \\ \text { Non-responders } & 5 & 29,4 & 5 & 41,7 & \end{array}$

Abbreviations: $C R$ complete response, $P R$ partial response, $P P$ pain progression, IP intermediate pain

(33.3\%) in the IMRT group had died, along with 11 patients (36.7\%) in the 3DCRT arm. Between 3 and 6 months, another 2 patients (10\%) died from tumor progression in the IMRT cohort, along with a further 7 patients (36.8\%) in the 3DCRT arm (Fig. 1). OS did not differ between groups $(p=0.187)$ (Fig. 2). The mean follow-up was 6.3 months (IQR 2.5-9.3) for both groups.

As compared to baseline, bone density became significantly higher at 3 and 6 months following IMRT by a median percentage of $24.8 \%$ and $33.8 \%(p<0.01$ for 3 months and $p=0.048$ for 6 months), respectively (Table 2). These figures in the 3DCRT cohort were $18.5 \%$ and $48.4 \%$ ( $\mathrm{p}<0.01$ for both), respectively. There were no statistical differences in bone density between IMRT and 3DCRT at $3(p=0.723)$ or 6 months $(p=0.341)$.

Subgroup evaluation of solely osteolytic lesions at 3 and 6 months showed no significant differences between groups ( $p=0.489$ and $p=0.377$ respectively) (Table 3 ). There were no differences between bone density changes in osteoblastic metastases in the IMRT and 3DCRT groups at 3 or 6 months ( $p=1.000$ for both) (Table 3). Subgroup evaluation of mixed lesions showed a significant difference $(p=0.037)$ at 6 months but not at 3 months $(p=0.256)$ (Table 3).

Bone density in unaffected vertebrae did not show substantial changes within groups at 3 and 6 months following RT (IMRT: $p=0.623$ and $p=0.167$, 3DCRT: $p=$ 0.934 and $p=0.147)$. There were also no significant differences between the IMRT and 3DCRT arms at $3(p=$ $0.574)$ or 6 months $(p=0.949)$.
Preexisting pathological fractures existed in 3.3\% patients in the IMRT arm vs. $13.3 \%$ in the 3DCRT group $(p=0.161)$ (Table 4). By 3 and 6 months, these numbers rose to $15.0 \%$ vs. $10.5 \%(p=0.676)$ and $16.7 \%$ vs. $16.7 \%$ $(\mathrm{p}=1.000)$, respectively. No pathological fractures in either group required salvage surgical intervention.

Pain assessment, using VAS scoring, was similar between cohorts at baseline $(p=0.882)$ and immediately following RT $(p=0.075)$. Although the IMRT arm showed improved pain response at 3 months $(\mathrm{p}=0.037)$, this was not observed at 6 months $(p=0.430)$. There were also no differences in neuropathic pain at $3(p=0.946)$ or $6(p=0.661)$ months. Using Chow criteria, pain response was statistically similar at both $3(p=0.395)$ and $6(p=0.732)$ months (Table 5). At 3 months, $70.0 \%$ of patients that underwent IMRT were categorized as responders, as compared to $47.4 \%$ in the 3DCRT arm $(p=0.151)$; these numbers at 6 months were $70.8 \%$ and $58.3 \%$, respectively $(p=0.494)$.

There were no differences in the pattern of recorded OMED consumption between groups at both 3 and 6 months after RT (Fig. 3).

\section{Discussion}

This prespecified secondary evaluation of a prospective randomized trial is the first to investigate the impact of image-guided IMRT on bone density as compared to 3DCRT. No differential effects on bone density or other secondary endpoints were expected between IMRT and 3DCRT techniques at the time of study creation. The significant rises in bone density after 3 and 6 months, along with pathologic fracture rates and pain response, were similar in both cohorts.

Despite rightful concerns regarding its cost-effectiveness for palliative vertebral irradiation, IMRT is an attractive option in part owing to the ability to perform simultaneous integrated boosting (SIB). This refers to allowing multiple target volumes to receive different doses per fraction, while maintaining the same total number of fractions. Although no patient in this trial received SIB, this topic will be better understood following maturation of the IRON-2 trial, which consists of four arms: 20 Gy in 5 fractions (with or without SIB to 30 Gy in 5 fractions) and 30 Gy in 10 fractions (with or without SIB to 40 Gy in 10 fractions) [8]. Evaluating bone density in such instances will be essential to evaluate whether higher fractional doses are safe from the bone density standpoint as well.

From these data, it was noteworthy that baseline bone density was higher in the IMRT arm $(p=0.037)$. Although numerically higher at 3 months as well (median 419 versus 300 ), this did not reach statistical significance $(p=0.232)$, likely owing to the lower sample sizes available at 3 months. Nevertheless, 6 month values were numerically comparable. Moreover, the relative magnitude of bone density change at 3 and 6 months was also 

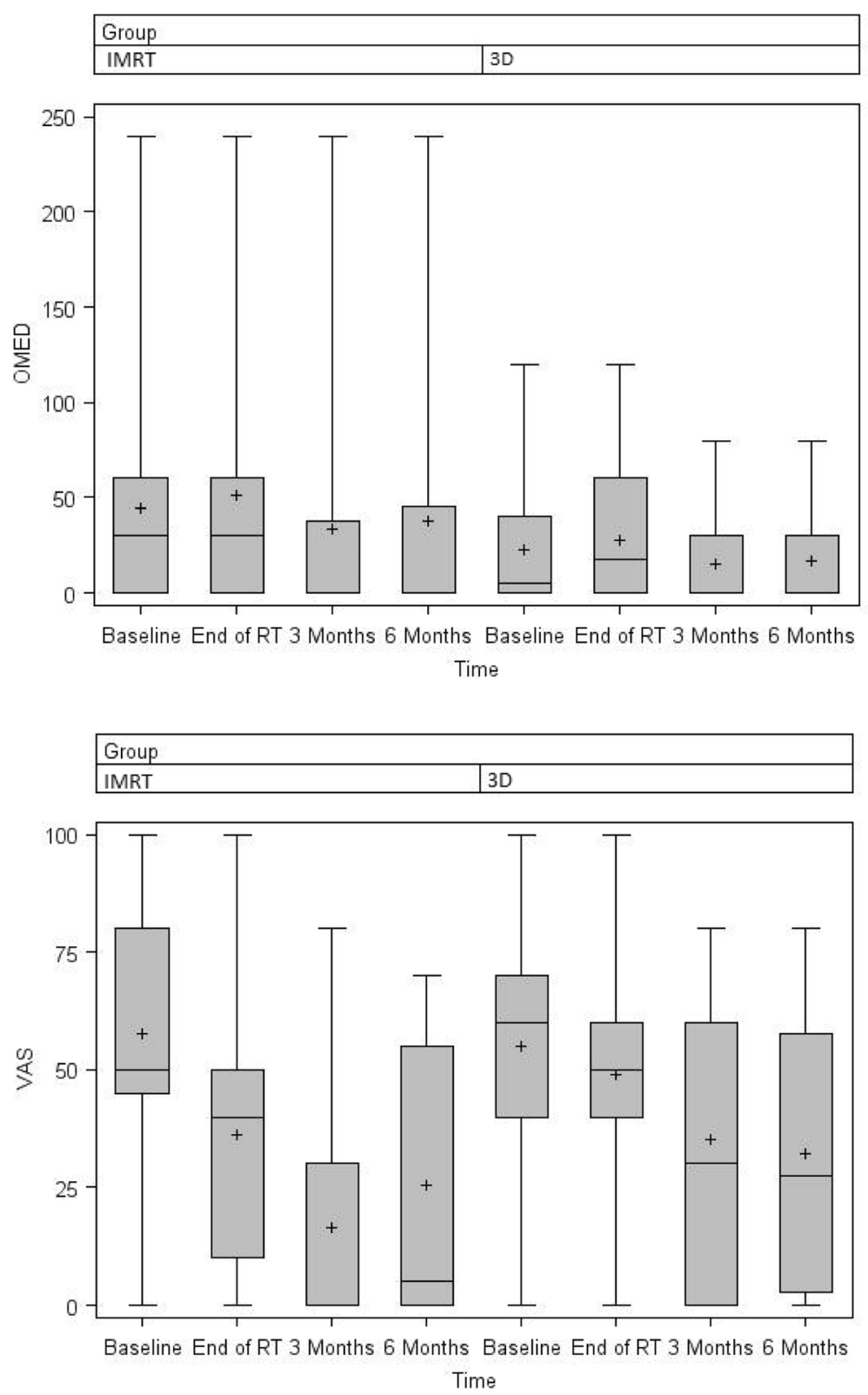

Fig. 3 OMED and VAS of both groups at measured points

numerically and statistically comparable between both groups. This implies that components of bone density changes specifically related to RT are likely similar between both cohorts.

What is less clear are the findings related to mixed osteolytic-osteoblastic lesions at 6 months. The threemonth values were not significantly different between cohorts, nor were those for purely osteolytic or osteoblastic metastases. As such, these analyses with clearly small sample sizes may not provide robust conclusions in this subgroup of patients. Additionally, only a few previous studies included mixed or osteoblastic metastases. In contrast to our results, Eggermont et al. did not observe any bone density changes in mixed proximal femur lesions after 4 and 10 weeks [9]. This could possibly result from an earlier date of collection and lower doses (prescription up to 20 or $24 \mathrm{~Gy}$ ). In line with these results, less remineralization in the extremities was reported by Rieden and coauthors [10].

Wachenfeld et al. reported an increase in CT density in osteolytic metastases to approximately $150 \%$ of the initial value at 3 months after multi-fraction irradiation [11]. Koswig and Budach showed improvement of bone density in osteolytic metastases by $173 \%$ at 6 months 
after multi-fraction irradiation [12]. In this trial, however, the differences were $20.3 \%$ and $9.3 \%$ in the IMRT and 3DCRT arms, respectively, at 3 months; 6-month values were $-0.8 \%$ and $27.7 \%$, respectively. There are several causes of these discrepancies, including the specific patient population, histology, size of metastases and several other factors.

The improved pain response based on VAS in the IMRT group ( $p=0.037)$ at 3 months could have been from a greater use of hormonal therapy in metastatic prostate carcinoma. That being said, potential imbalances in antiosteoresorptive therapies are an unlikely cause of the findings herein, as densities of unaffected vertebrae yielded no differences between groups. Rief et al. studied the impact of resistance training concomitantly with conventional multi-fraction 3DCRT on bone density in a randomized controlled study and found no significant differences in the uninvolved spine [13]. Therefore, it has been suggested that bisphosphonates may not exert decisive effects in this circumstance.

Despite the randomized design and standardized evaluation of bone density and recording of all pathological structures, several limitations must be noted. In addition to the lower sample size and shorter follow-up, robust conclusions based on statistical comparisons cannot be made, as partially discussed above. Second, a possible methodological weakness in our study was the lack of a control group. Third, many patients did not receive concurrent radiotherapy and chemotherapy, so the relationship of systemic therapy on bone density changes cannot be entirely excluded [14-18]. Fourth, some participants received immunotherapy and prior or subsequent radiotherapy to other distant metastases. The abscopal effect in this setting has been sporadically described but not sufficiently clarified $[19,20]$, but our study did not investigate this causality. Fifth, few studies can entirely account for other factors influencing bone density such as diet, particular medications, or vitamin supplementation. There may also be heterogeneity in this population given the specific location of vertebral metastases (e.g. vertebral body versus lamina/pedicle) as well as degree of soft tissue extension. Although these may limit applicability to other studies, larger randomized data are recommended to validate these results.

\section{Conclusions}

This prespecified secondary evaluation of a prospective randomized trial is the first to investigate the impact of image-guided IMRT on bone density as compared to 3DCRT. The significant rises in bone density after 3 and 6 months, along with pain response and pathologic fracture rates, were similar in both cohorts. Future randomized investigations with larger sample sizes are recommended.

\section{Abbreviations}

CR: Complete response; CT: Computed tomography; CTV: Clinical target volume; 3DCRT: conventional 3D conformal radiotherapy; EBRT: external body radiotherapy; Gy: Gray; HU: Hounsfield unit; IMRT: Intensity-modulated radiotherapy; kV: kilo-voltage; MV: Megavoltage; SIB: simultaneous integrated boost; OAR: Organ at risk; OMED: oral equivalent morphine dose; OS: Overall survival; PP: Progression pain; PR: Partial response; PTV: Planning target volume; QoL: Quality of life; RT: Radiotherapy; SBRT: Stereotactic body radiation therapy; IP: Intermediate pain; SRS: Stereotactic radiosurgery; VAS: Visual analog scale; VCF: Vertebral compression fracture; VMAT: Volumetric modulated arc therapy

\section{Acknowledgements}

We thank the German Bone Cancer Research Group Members for their great effort.

\section{Funding}

Not applicable.

\section{Availability of data and materials}

The data used in this analysis are from publications available in the public domain.

\section{Authors' contributions}

$H R$ and JD developed and planned this trial. TB was responsible for statistical considerations. TS, W, RF, IS, NHN, TB, RES and HR performed the examinations and RT supervisions. TW (radiologist) planned the diagnostic examination of computed tomography. HR and TS made the data collection. All authors read and approved the final manuscript.

\section{Ethics approval and consent to participate}

The Independent Ethics Committee of Heidelberg University (Nr. S-238/2016).

\section{Consent for publication}

Not applicable.

\section{Competing interests}

The authors declare that they have no competing interests.

\section{Publisher's Note}

Springer Nature remains neutral with regard to jurisdictional claims in published maps and institutional affiliations.

\section{Author details}

${ }^{1}$ University Hospital of Heidelberg, Department of Radiation Oncology, Im Neuenheimer Feld 400, 69120 Heidelberg, Germany. ${ }^{2}$ Department of Radiation Oncology, Allegheny General Hospital, Pittsburgh, PA, USA. ${ }^{3}$ Heidelberg Institute of Radiation Oncology (HIRO), Im Neuenheimer Feld 280, 69120 Heidelberg, Germany. ${ }^{4}$ University Hospital Zurich, Department of Radiation Oncology, Raemistrasse 100, 8091 Zurich, Switzerland. ${ }^{5}$ University Hospital of Heidelberg, Department of Medical Biometry, Im Neuenheimer Feld 305, 69120 Heidelberg, Germany. ${ }^{6}$ University Hospital of Freiburg, Department of Radiation Oncology, Robert-Koch-Strasse 3, 79106 Freiburg, Germany.

Received: 13 April 2018 Accepted: 22 October 2018

Published online: 30 October 2018

References

1. Wong DA, Fornasier VL, MacNab I. Spinal metastases: the obvious, the occult, and the impostors. Spine. 1990;15(1):1-4.

2. Sze WM, Shelley M, Held I, Mason M. Palliation of metastatic bone pain: single fraction versus multifraction radiotherapy - a systematic review of the randomised trials. Cochrane Database Syst Rev. 2004;2:CD004721.

3. McQuay HJ, Collins SL, Carroll D, Moore RA. Radiotherapy for the palliation of painful bone metastases. Cochrane Database Syst Rev. 2000;2:Cd001793.

4. Meyerhof E, Sprave T, Welte SE, Nicolay NH, Forster R, Bostel T, Bruckner T, Schlampp I, Debus J, Rief H. Radiation-induced toxicity after image-guided and intensity-modulated radiotherapy versus external beam radiotherapy for patients with spinal bone metastases (IRON-1): a study protocol for a randomized controlled pilot trial. Trials. 2017;18(1):98. 
5. Chow E, Wu JS, Hoskin P, Coia LR, Bentzen SM, Blitzer PH. International consensus on palliative radiotherapy endpoints for future clinical trials in bone metastases. Radiother Oncol. 2002;64(3):275-80.

6. Marks LB, Yorke ED, Jackson A, Ten Haken RK, Constine LS, Eisbruch A Bentzen SM, Nam J, Deasy JO. Use of normal tissue complication probability models in the clinic. Int J Radiat Oncol Biol Phys. 2010;76(3 Suppl):S10-9.

7. Sprave T, Verma V, Forster R, Schlampp I, Bruckner T, Bostel T, Welte SE, Tonndorf-Martini E, El Shafie R, Nicolay NH, et al. Radiation-induced acute toxicities after image-guided intensity-modulated radiotherapy versus threedimensional conformal radiotherapy for patients with spinal metastases (IRON-1 trial) : First results of a randomized controlled trial. Strahlentherapie und Onkologie. 2018. https://doi.org/10.1007/s00066-018-1333-z Epub ahead of print

8. Sprave T, Welte SE, Bruckner T, Forster R, Bostel T, Schlampp I, Nicolay NH, Debus J, Rief H. Intensity-modulated radiotherapy with integrated-boost in patients with bone metastasis of the spine: study protocol for a randomized controlled trial. Trials. 2018;19(1):59.

9. Eggermont F, Derikx LC, Verdonschot N, Hannink G, Kaatee R, Tanck E, van der Linden YM. Limited short-term effect of palliative radiation therapy on quantitative computed tomography-derived bone mineral density in femora with metastases. Advances in radiation oncology. 2017;2(1):53-61.

10. Rieden K, Adolph J, Lellig U, Zum Winkel K. The radiotherapeutic effect on bone metastases in relation to the frequency of metastases, sites of metastases and histology of the primary tumor. Strahlentherapie und Onkologie. 1989;165(5):380-5.

11. Wachenfeld I, Sanner G, Bottcher HD, Kollath J. The remineralization of the vertebral metastases of breast carcinoma after radiotherapy. Strahlentherapie und Onkologie. 1996;172(6):332-41.

12. Koswig S, Budach V. Remineralization and pain relief in bone metastases after after different radiotherapy fractions (10 times 3 Gy vs. 1 time 8 Gy). A prospective study. Strahlenther Onkol. 1999;175(10):500-8.

13. Rief H, Omlor G, Akbar M, Welzel T, Bruckner T, Rieken S, Haefner MF, Schlampp I, Gioules A, Habermehl D, et al. Feasibility of isometric spinal muscle training in patients with bone metastases under radiation therapy - first results of a randomized pilot trial. BMC Cancer. 2014;14:67.

14. Strang P, Bergqvist J. Does palliative chemotherapy provide a palliative effect on symptoms in late palliative stages? An interview study with oncologists. Acta oncologica. 2017;56(10):1258-64.

15. Daimon T, Kosaka T, Oya M. A metastatic castration resistant prostate cancer patient with multiple bone metastases has durable biochemical and radiological response to docetaxel chemotherapy. American journal of clinical and experimental urology. 2016;4(2):28-31.

16. Nieder C, Haukland E, Mannsaker B, Norum J. Impact of intense systemic therapy and improved survival on the use of palliative radiotherapy in patients with bone metastases from prostate cancer. Oncol Lett. 2016; 12(4):2930-5.

17. Steinauer K, Huang DJ, Eppenberger-Castori S, Amann E, Guth U. Bone metastases in breast cancer: frequency, metastatic pattern and non-systemic locoregional therapy. Journal of bone oncology. 2014;3(2):54-60.

18. Groenen KH, Pouw MH, Hannink G, Hosman AJ, van der Linden YM, Verdonschot N, Tanck E. The effect of radiotherapy, and radiotherapy combined with bisphosphonates or RANK ligand inhibitors on bone quality in bone metastases. A systematic review. Radiother Oncol. 2016; 119(2):194-201.

19. Leung HW, Wang SY, Jin-Jhih H, Chan AL. Abscopal effect of radiation on bone metastases of breast cancer: a case report. Cancer Biol. Ther. 2018; 19(1):20-4.

20. Brix N, Tiefenthaller A, Anders H, Belka C, Lauber K. Abscopal, immunological effects of radiotherapy: narrowing the gap between clinical and preclinical experiences. Immunol Rev. 2017;280(1):249-79.

\section{Ready to submit your research? Choose BMC and benefit from:}

- fast, convenient online submission

- thorough peer review by experienced researchers in your field

- rapid publication on acceptance

- support for research data, including large and complex data types

- gold Open Access which fosters wider collaboration and increased citations

- maximum visibility for your research: over $100 \mathrm{M}$ website views per year

At BMC, research is always in progress.

Learn more biomedcentral.com/submissions 